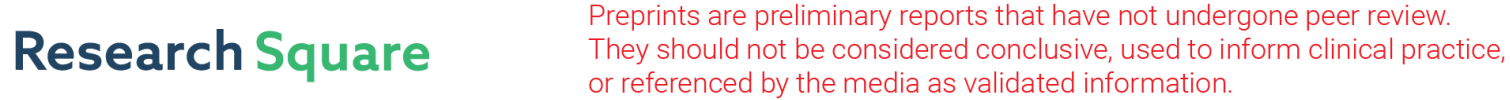 \\ Statistical Characterization of Airplane Delays
}

\section{Evangelos Mitsokapas}

Queen Mary University of London

Benjamin Schäfer ( $\sim$ b.schaefer@qmul.ac.uk)

Queen Mary University of London

Rosemary Harris

Queen Mary University of London

Christian Beck

Queen Mary University of London

\section{Research Article}

Keywords: aviation industry, globally connected economy, COVID-19 pandemic

Posted Date: December 29th, 2020

DOI: https://doi.org/10.21203/rs.3.rs-133115/v1

License: (c) (1) This work is licensed under a Creative Commons Attribution 4.0 International License.

Read Full License

Version of Record: A version of this preprint was published at Scientific Reports on April 12th, 2021. See the published version at https://doi.org/10.1038/s41598-021-87279-8. 


\title{
Statistical Characterization of Airplane Delays
}

\author{
Evangelos Mitsokapas, ${ }^{1}$ Benjamin Schäfer,, , Rosemary Harris, ${ }^{1}$ and Christian Beck ${ }^{1}$ \\ ${ }^{1}$ School of Mathematical Sciences, Queen Mary University of London, London E1 4NS, United Kingdom \\ The aviation industry is of great importance for a globally connected economy. Customer sat- \\ isfaction with airlines and airport performance is considerably influenced by how much flights are \\ delayed. But how should the delay be quantified with thousands of flights for each airport and \\ airline? Here, we present a statistical analysis of arrival delays at several UK airports between 2018 \\ and 2020. We establish a procedure to compare both mean delay and extreme events among airlines \\ and airports, identifying a power-law decay of large delays. Furthermore, we note drastic changes \\ in plane delay statistics during the COVID-19 pandemic. Finally, we find that delays are described \\ by a superposition of simple distributions, leading to a superstatistics.
}

\section{INTRODUCTION}

The aviation industry was a rapidly growing sector until recently, prior to the current COVID-19 pandemic. Economic growth led to higher average yearly distances travelled, as well as higher air traffic volumes, robustly observed among several regions worldwide until 2019 [1, 2. But both the ongoing pandemic [3] and also the push towards more renewable options in aviation [4] may induce a considerable change in the industry in the future. This makes the industry a very interesting object to study as it transforms.

As a passenger, an important benchmark for evaluating travel options, e.g. in terms of airports, airlines or even modes of transportation (train vs plane) is the punctuality of each option. In particular, flight delays severely decrease customer satisfaction and might lead to customers choosing a different airport or airline, especially if a timely arrival is urgently required [5]. Generally, it is important to quantitatively understand delay-risks both in terms of the expectation values but also in terms of the extreme events, i.e. quantifying how likely a very early or very late arrival is.

The study of delays in aviation is already an active field of research. Previous, simple, investigation frameworks to classify and categorize delays have been proposed [6] but mostly relied on mean values. In other cases, stochastic models of plane delays [7] were developed without considering the corresponding probability distributions or assuming simple Normal or Poisson distributions [8]. More recent work also includes the application of machine learning techniques to aviation data, e.g. via recurrent neural networks [9]. One problem of any data-driven approach is that many articles on aviation research solely rely on proprietary data: In a recent review investigating 200 research articles, $68 \%$ were based on proprietary data [10. Hence, to enable the broader applicability of machine learning applications, more publicly available data are still required.

To quantify delay statistics, we will go beyond the oft-used averages of delays [6] and instead investigate the entire probability density function, as it encodes the full range of delay information, from highly negative delays (i.e. flights arriving significantly earlier than their scheduled arrival time) to severely positively delayed flights. To explain the emergence of heavy tails, i.e. extreme deviations from the mean, we will utilize superstatistical modelling [11. Such an approach has been successfully applied in transport before, for modelling train delays [12]; it has also attracted recent interest when describing fluctuations in the energy system [13 and air pollutant concentrations 14 and it has been extended to the general framework of diffusing diffusivities in nonequilibrium statistical physics and biologically inspired physics [15].

In this article, we present new data collected from 2018 to 2020 at several UK airports, with a particular focus on Heathrow, being the most important international hub in the UK. The data 
were publicly available from the arrival information of each airport, given out on their websites each day but had to be collected and processed for further usage. We analyse the full probability density of delay distributions and introduce certain performance indices to describe these distributions, such as the mean delay, the exponential decay rate of negative delays, and the power-law exponent of large delays. These indices are then compared for the different UK airports and the different airlines operating at these airports, to understand the main features of the delay statistics in a more systematic way. Finally, we deal with a theoretical model to explain features of the delay statistics. We show that the power law of large positive delays can be linked to a superposition of exponential delays with a varying decay parameter, in a superstatistical approach. On the contrary, negative delays (early arrivals) do not exhibit any power laws but simply behave in an exponential way, with extremely early arrivals exponentially unlikely. Throughout this article, we assume that passengers prefer to arrive as early as possible, i.e. with as little positive and as much negative delay as possible.

\section{NEW DATA}

We collected flight details from a number of different airports. For the purposes of this article, we have taken into consideration the top five UK airports, in order of passenger traffic [16, namely: London Heathrow Airport (LHR), London Gatwick Airport (LGW), London Luton Airport (LTN), London Stansted Airport (STN) and Manchester Airport (MAN). For a period of time lasting between Autumn 2018 and Spring 2019, we collected a combined total of approximately twohundred and twenty thousand $\left(2.2 \times 10^{5}\right)$ flight-arrivals from all five airports mentioned above. Furthermore, we continued collecting flight-information from London Heathrow during the 2020 COVID-19 pandemic, to illustrate the effect the lockdown had on the delay distribution. For each flight, we recorded the airline company operating the flight along with the corresponding flight number, departure and arrival airports, as well as scheduled and actual landing times. The delay is then computed simply as the difference between an aircraft's scheduled arrival time and its actual arrival time. We made all collected data publicly available. For details of the data processing and availability, see Methods.

The main body of our data (about 85\%) is sourced from London Heathrow, making it the chief focus of our analysis simply due to its size. London Heathrow is an international airport operating flights of 80 different airlines in total, which fly to 84 different countries around the world, as of 2019 [16]. Of course, in addition there are domestic flights within the UK. The passenger nationalities are $48 \%$ European and UK and $52 \%$ from the rest of the world. It is the busiest airport in Europe by passenger traffic [16].

The empirical probability density function (PDF) of all delays is a key characteristic to monitor, see Fig. 1 for all Heathrow delays. There, we compare the data collected from 2018 to 2019 with more recent data collected during the 2020 COVID-19 pandemic (during the first lockdown in Spring to Summer 2020), which led to a drastic reduction in air transport 17, 18. There are two interesting observations: Firstly, the delay statistics under COVID-19 are shifted to the left, indicating overall smaller delays (including more negative delays); secondly, the general shape of the distribution does not change drastically. In particular, we observe a fast decay of the PDF of negative delays on the left side and a much slower decay of the PDF on the right side for positive delays. In the following sections, we will analyse this behaviour in much more detail.

\section{QUANTIFYING DELAY STATISTICS}

Starting from a histogram of the flight delays, we derive three indices/measures to quantify flight delay distributions: Mean delay, exponent of left exponential and power-law exponent of right $q$ exponential, as explained below in detail. We will use the LHR data previous to any COVID-19 influence as our main example. 


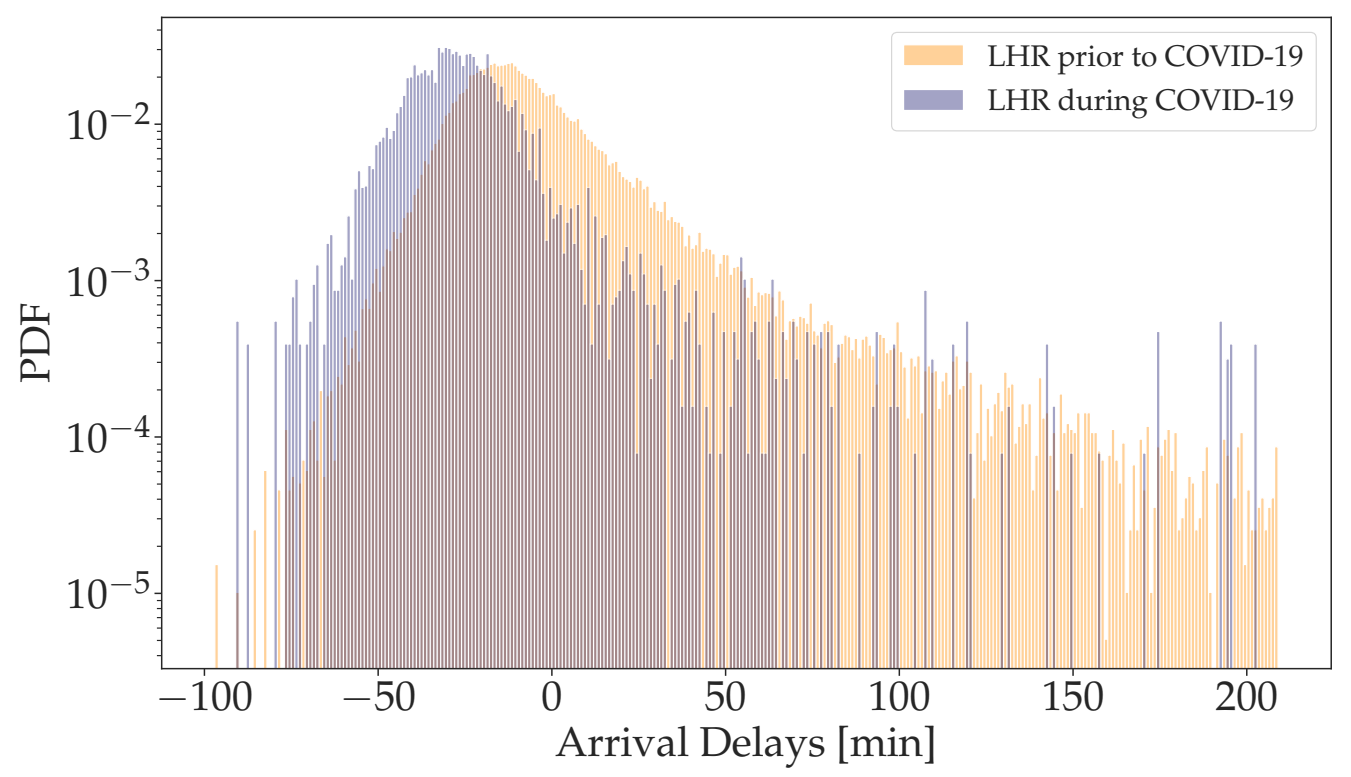

FIG. 1. Flight delays follow a broad distribution with large negative and positive delays. We display LHR delay histograms prior to and during the COVID-19 pandemic, both normalized. As the COVID-19 LHR data set is significantly smaller in size, compared to the regular LHR data set, it contains many gaps, where no data were recorded. The COVID-19 data set is significantly shifted towards the left (smaller delays) as compared to the pre-pandemic time.

As a first step, we split the full histogram at its peak value into two histograms, a left flank of dominantly negative delays and a right flank of dominantly positive delays, see Fig. 2. Based on the shape of the empirical distributions, we use exponentials and $q$-exponentials as fitting functions, see also Methods for details.

The left flank is observed to be well approximated by an exponential function of the form

$$
p\left(t_{L} ; \lambda\right)=\lambda e^{-\lambda t_{L}}, \lambda>0,
$$

where $t_{L}$ are the normalized arrival delays on the left flank, see Methods for details. The exponent $\lambda$ here quantifies the exponential decay of the probability of early arrivals. Therefore, a large $\lambda$ implies that few flights arrive very early while a small $\lambda$ indicates that very large negative delays are observed. Hence, typically, we assume a small $\lambda$ to be desirable.

The right flank of the delay distribution obeys a power law, i.e. a slow decay of $p \sim t^{\nu}$, with $\nu$ negative. To quantitatively describe the right flank, we use a $q$-exponential function [19] of the form

$$
p\left(t_{R} ; q, \lambda_{q}\right)=(2-q) \lambda_{q}\left[1+(q-1) \lambda_{q} t_{R}\right]^{\frac{1}{1-q}},
$$

where $t_{R}$ are the normalized arrival delays on the right flank, see Methods for details. The powerlaw exponent, i.e., the rate at which the probability density decays for high (positive) delay values, is given by $\nu:=1 /(1-q), 1<q<2$. Note that the scale parameter $\lambda_{q}>0$ is relevant for the precise fit but will not be used as a delay index as its influence on the delay distribution is limited. Contrary to the left-flank exponential decay, the absolute values of the right-flank power law exponent $\nu$ should be as large as possible. Large (absolute) values of $\nu$ imply a rapid decay of positive delays, i.e. fewer extreme events of very delayed arrivals.

Finally, we note that the two flanks describe the tails of the distribution well but overestimate the peak, i.e. the most likely value, see Fig. 3. Hence, we complement the two previous fits by using the mean delay $\mu$ as a third index. Since we assume that passengers wish to arrive as early as 

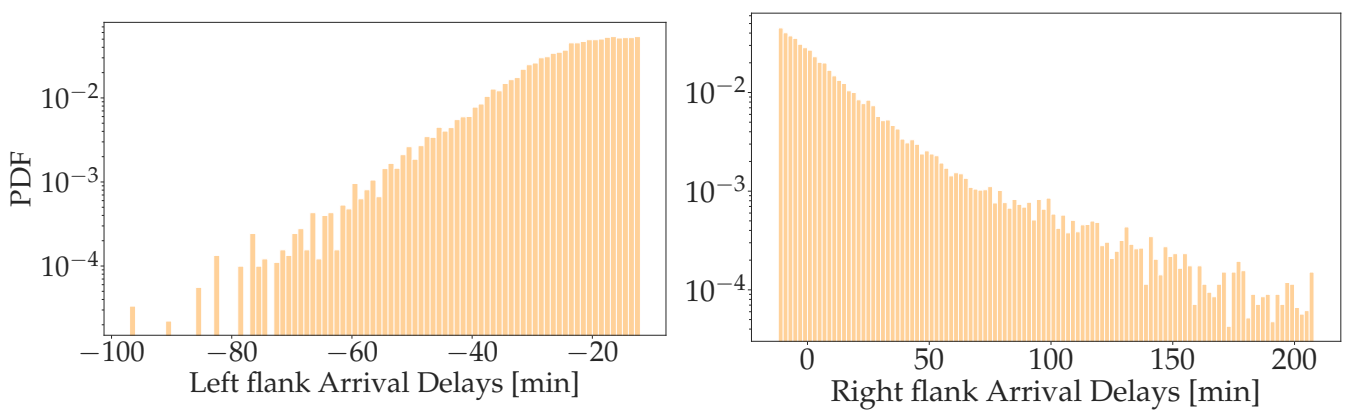

FIG. 2. Splitting the full distribution at the peak leads to two easier-to-fit flanks. Left: Negative delays decay approximately linearly in the log-scale and thereby suggest an exponential fit (1) Right: Positive delays display substantial heavy tails and thereby suggest the usage of a $q$-exponential function (2).

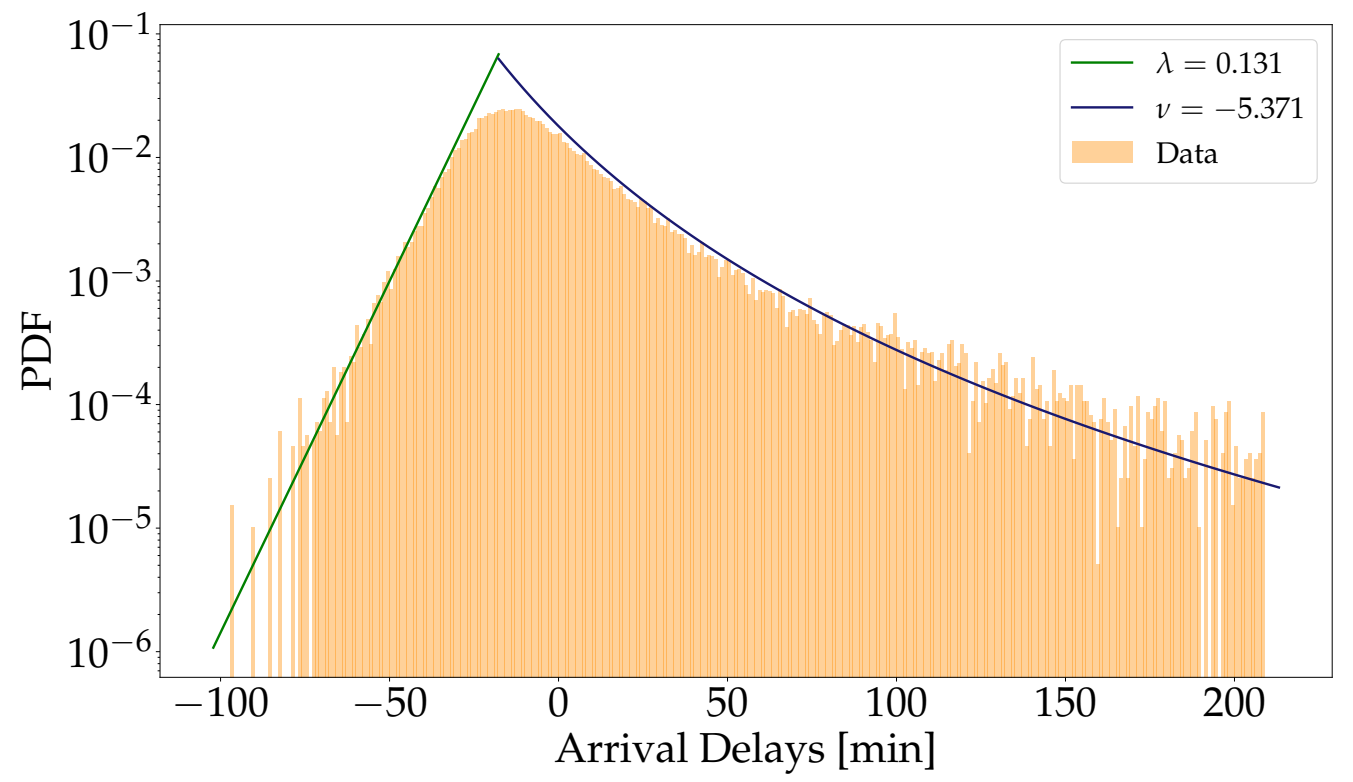

FIG. 3. Exponential (green) and $q$-exponential (blue) theoretical distributions capture the empirical distribution. The fits are obtained via the MLE method, see Methods for fitting details. To complement the over-estimated "peak" (tent-like shape) we introduce the mean delay $\mu$ index.

possible, the mean $\mu$ should be as small (or as negative) as possible. In the case of LHR, the three delay indices that we introduced are $\lambda=0.131, \mu=-5.06$ and $\nu=-5.371$. We also introduce a continuous smooth fitting function for the full range in Section VI

Note that the mean value $\mu$ can be easily manipulated by airline companies by scheduling flight arrival times later then actually needed, hence always causing a negative mean delay, which may artificially improve their performance. On the contrary, the tail behavior truthfully represents the extreme event statistics for both positive and negative delays and cannot be easily manipulated by the operators. 


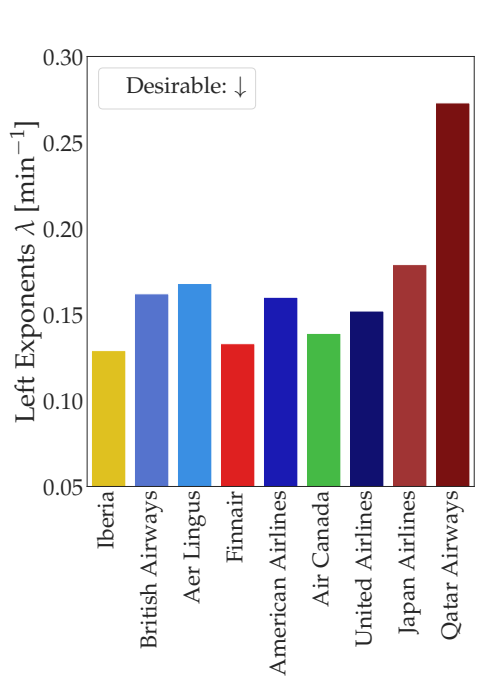

Airlines covering long-distance flights
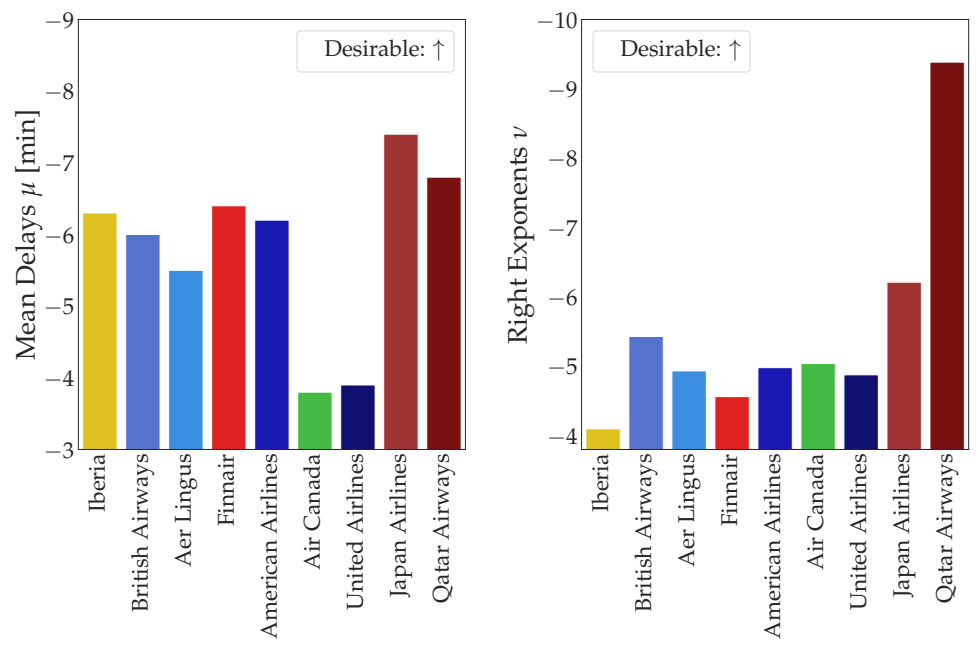

FIG. 4. International airlines appear to differ substantially in their three delay indices. We plot the leftside (negative) delay exponential decay, right-side (positive) power-law delay decay and the mean delay. Arrows indicate whether a small or large value is desirable.

\section{COMPARISON OF AIRPORTS AND AIRLINES}

We here use the previously developed framework to quantify and compare delay statistics for different airlines and airports. Intuitively, we expect that long-distance flights would, on average, yield more extreme early or late arrivals, compared to the corresponding short-distance ones. Thus, we distinguish between short-distance airlines, covering mostly domestic and European destinations, and airlines that include long-distance, international destinations, as well as destinations within Europe. We first compute the three indices $\lambda, \mu, \nu$ for each of those airline groups and then compare full airport statistics, aggregating all airlines.

There are several factors impacting the delay distribution for each airport or airline: Airline policies, flight routes, technical defects or issues with documentation contribute to $27 \%$ of all delays [20]. Specifically, overseas flights are more sensitive to wind (head wind or tail wind), as well as unstable weather conditions (storms, fog) and military exercises. Airlines operating international flights, as illustrated in Fig. 4 . exhibit considerable variations in their flight delay indices. Note that while a low left exponent $\lambda$ may be regarded as a desirable property (flights often arrive very early), the mean $\mu$ and the right exponent $\nu$ should definitely be as small as possible (low mean delay and few very late arrivals). Since both quantities tend to be negative, their absolute values should be large. Comparing the airlines, we observe a "grouping" behaviour for some of the carriers. On the one hand, airlines having a blend between short-distance (e.g. domestic or EU) and overseas destinations, such as Iberia, British Airways (BA), Aer Lingus and Finnair, appear to follow a similar trend for each index. On the other hand, airlines that do not possess such a spread of destinations tend to perform well only in some of the indices. As an illustrative example we choose Air Canada and United Airlines: Although both their left and right exponents are in a similar range to the other airlines, their mean delays are substantially less negative than those of their competitors.

Characterization of short-distance flights shows a strong grouping of the delay behavior for some airlines. As seen in Fig. 5, comparison of five of the largest low-cost domestic and European providers, reveals a systematic similarity between Wizz Air, easyJet and Ryanair. All three airlines manage to perform well in the left exponent metric, minimizing early arrivals, while they maintain an acceptable negative average delay (with easyJet performing worst here). Again, they follow a 
Airlines not covering long-distance flights
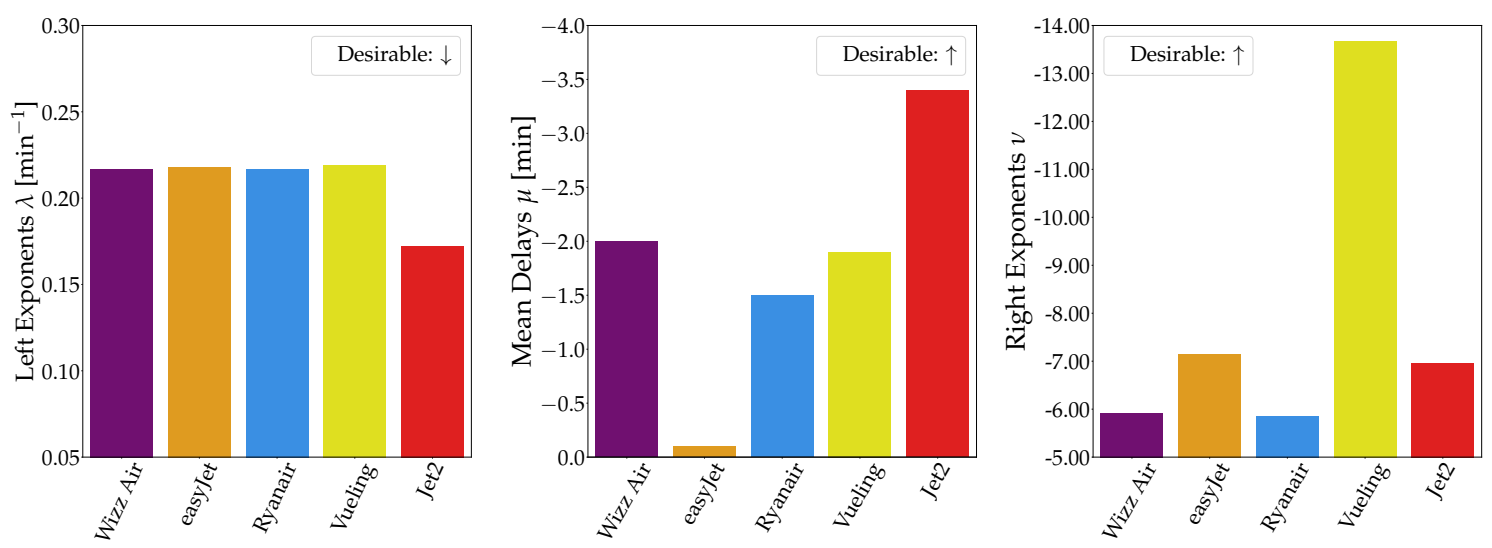

FIG. 5. Delay indices for low-cost airlines not covering long-distance flights. Wizz Air, easyjet, Ryanair and Vueling share the largest $\lambda$ index (early arrivals). Jet2 has the smallest mean delay $\mu$ and Vueling is characterized by the smallest $\nu$ index (late arrivals).

similar right-exponent trend, translated to a certain number of overall late arrivals. Furthermore, Jet2 outperforms all other short-distance airlines in $\lambda$ left-exponents and mean delays. Finally, Vueling resembles Wizz Air and Ryanair values in the $\lambda$ and $\mu$ metrics but seems to have less late arrivals as per its high right exponent $\nu$.

Comparing the long distance airlines with the short-distance ones, we notice some differences: Airlines covering long distances tend to display lower (more desirable) left exponents as well as higher negative mean delays. Meanwhile, the right exponent behavior is similar between the two groups with Vueling and Qatar Airlines as the "outliers" in their respective category. Whether this behavior is due to company policies or flight distance remains a question for future research.

Studying the indices for individual airports yields interesting insights as well. Airports populated by airlines flying mainly to domestic and EU destinations, such as LTN and STN, score fairly well in both early and late arrivals, with an approximately net zero mean delay, see Fig. 6. On the one hand, STN is characterized by the minimum $\lambda$ value, showing the best performance in early arrivals in the group of airports, while LTN attains an acceptable value. On the other hand, it can be seen that LTN scores the second best $\nu$ value while STN lies very slightly above the median group $\nu$. Interestingly, mean delays at MAN airport are net positive, contrary to LHR and GAT where arrivals are scheduled in such a way that the mean delay is negative. Furthermore, MAN seems to not perform that well in the early arrivals index, having the worst score, but does outperform all the rest of the airports when compared under the extreme positive delays. International airports such as LHR and GAT (with the exception of LHR COVID-19) tend to cluster around similar values for all delay indices.

LHR during the COVID-19 pandemic outperforms all airports on the mean delay index by a large margin. The reason behind this is that the dramatic reduction of flight traffic worldwide saw many flights arriving too early. Interestingly, the left exponent, i.e. the decay of early arrivals, did not change substantially, compared to business-as-usual since the shape of the delay distribution on the left did not change much but was only shifted to more negative values. The right flank behaves quite differently: Both business-as-usual and LHR during the COVID-19 pandemic, recorded heavily delayed flights, which arrived more than 3 hours late (see also Fig. 1). The right index reveals the likelihood of these extreme events. In the case of LHR under COVID-19, the low mean delay suggests early arrival but the extreme events are still present and hence the right exponent reveals this poor performance. 

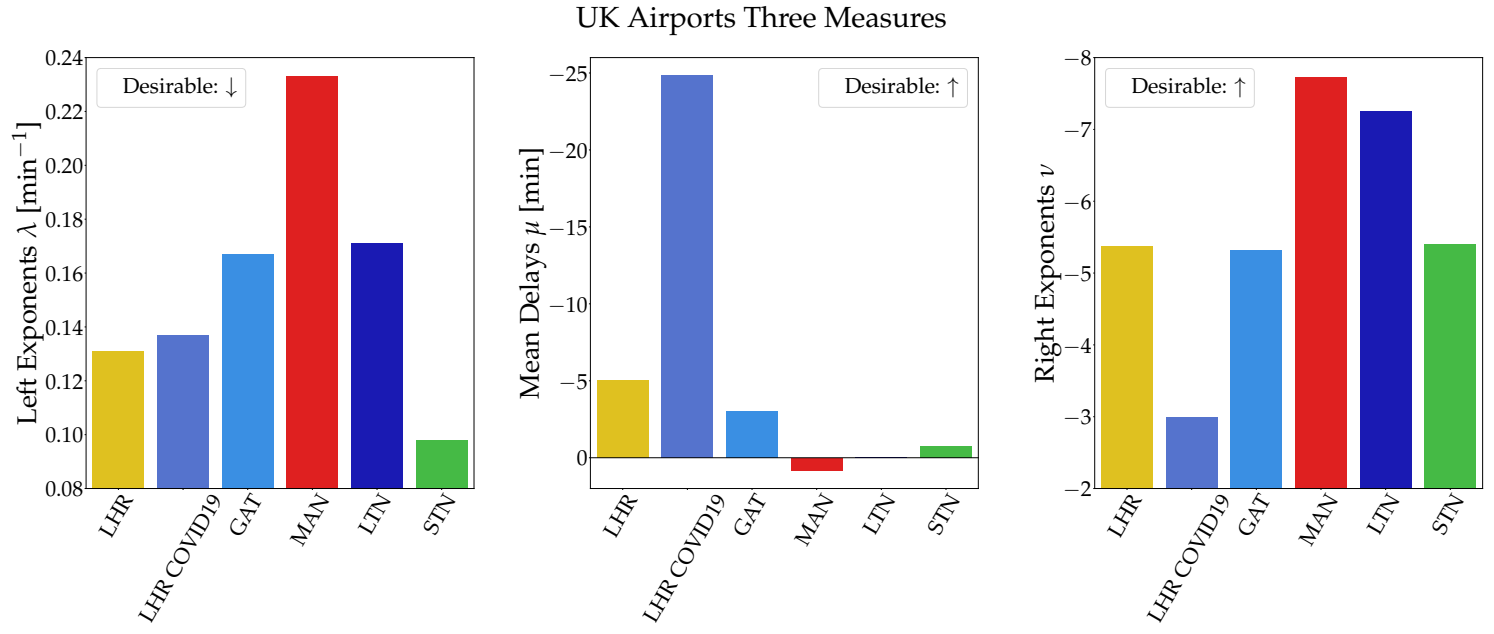

FIG. 6. Airports appear to differ substantially in the three delay metrics. Airports that serve mostly domestic and European destinations, such as LTN and STN, behave differently from international airports such as LHR, GAT and MAN.

\section{SUPERSTATISTICAL MODELLING OF DELAYS}

As we have seen previously, the right flank of the delay statistics exhibits heavy tails and is well-described by a $q$-exponential. Let us now explore a potential explanation for this particular distribution by employing the framework of superstatistics [11, 21, 22. Superstatistics is relevant when an aggregated system (e.g. a long time series) displays heavy tails, but the system may then be disentangled into many smaller sub-parts (e.g. short time periods of the trajectory). These sub-parts then are no longer heavy-tailed but follow a simple local distribution, for example an exponential or a Gaussian. This idea has been successfully applied, for example, to train delays [12, electric power systems [13] and intermittent wind statistics [23].

Assuming for now that the right-flank delays are indeed $q$-exponentially distributed and follow a superstatistics, we should be able to observe "local" exponential densities, with a decay parameter $\lambda$. Superimposing all these $\lambda$, we get a $q$-exponential if the $\lambda$ themselves follow a $\chi^{2}$-distribution:

$$
f(\lambda)=\frac{1}{\Gamma\left(\frac{n}{2}\right)}\left(\frac{n}{2 \lambda_{0}}\right)^{\frac{n}{2}} \lambda^{\frac{n}{2}-1} e^{-\frac{n \lambda}{2 \lambda_{0}}} .
$$

Here $n$ denotes the number of degrees of freedom characterizing the fluctuations in $\lambda$ and $\lambda_{0}$ is the sample mean of $\lambda$. Indeed, choosing an adequate time scale to separate the trajectory (see next paragraph), the heavy tails of delay distributions vanish and instead the distributions are well described by simple exponential functions, see Fig. 7.

Let us explain how to extract the relevant time scale $T$ on which we locally observe exponential distributions. Since we know that an exponential distribution has a kurtosis of $\kappa_{\text {exponential }}=9$, we test time windows of different size $\Delta \tau$ and compute the local average kurtosis [11] as

$$
\bar{\kappa}(\Delta \tau)=\frac{1}{\tau_{\max }-\Delta \tau} \int_{0}^{\tau_{\max }-\Delta \tau} d \tau_{0} \frac{\left\langle(u-\bar{u})^{4}\right\rangle_{\tau_{0}, \Delta \tau}}{\left\langle(u-\bar{u})^{2}\right\rangle_{\tau_{0}, \Delta \tau}^{2}},
$$

where $\tau_{\max }$ is the length of the time series $u$ and $\bar{u}$ is the mean of the time series. We denote by $\langle\ldots\rangle_{\tau_{0}, \Delta \tau}$ the expectation formed for a time slice of length $\Delta \tau$ starting at $\tau_{0}$. For the LHR data, we show how to determine the long time scale $T$ in Fig. 8 ,

Next, let us carry out an important consistency check: As explained above, the mixing of numerous local exponential distributions with exponents following a $\chi^{2}$-distribution leads to a 


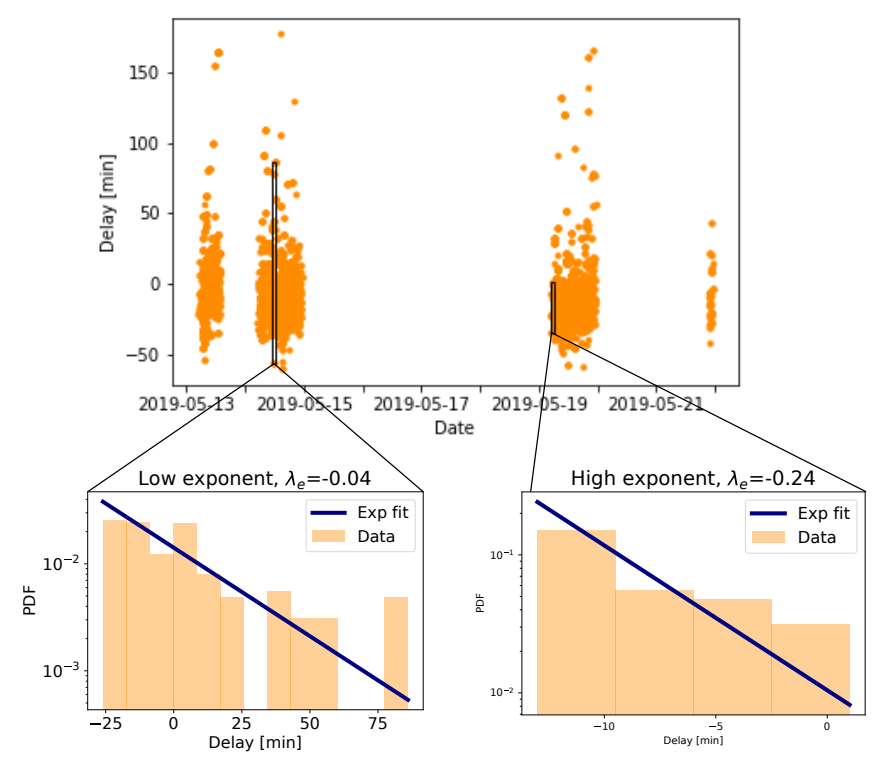

FIG. 7. We analyse the full time series of plane delays and extract a time window during which we observe locally exponential distributions. These local distributions can decay slowly or fast, i.e. the rate $\lambda$ is fluctuating.

$q$-exponential. Now, we can make a histogram of the $\lambda$-distribution and fit it with a $\chi^{2}$ - and an inverse $\chi^{2}$-distribution. Next, we derive the $q$-exponential from the fitted $\chi^{2}$-distribution and compare it with the direct fit of the $q$-exponential and the original data. This is illustrated in Fig. 9 .

We note that the empirical $\lambda$-distribution is slightly better fitted by an inverse $\chi^{2}$ - than a $\chi^{2}$-distribution, as also observed in other application areas [14, 24. Overall, the superstatistical description seems consistent, given the short time series of flight delays under consideration. The $q$ exponential derived from the $\chi^{2}$ tends overestimate the PDF at low values, which is understandable as we also exclude them for the fitting of the $q$-exponential via MLE (see methods). Still, the tail behavior of the $q$-exponential based on the $\chi^{2}$ matches the real data and the MLE fit nicely. This means the observed power laws of the right flank are essentially explained by a suitable superstatistics which describes changes in the microvariables on a time scale of $T \approx 1.5$ hours.

\section{CONNECTING THE FLANKS}

So far, we focused our attention on describing and fitting the tail aspects of the distribution, namely the left, approximately exponential, flank and the right, approximately $q$-exponential, flank. Both these functions combined overestimate the peak of the distribution and hence, we also included the mean delay as the final metric in our framework. Now, let us consider how the two tail distributions could be merged in one smooth-fitting function.

First, we note that the so far mostly ignored central part of the delay distribution might be approximated by a Gaussian distribution, based on the parabola shape in the log-scale plots. We use this insight to propose the following continuous fitting function

$$
p(t)=\left\{\begin{array}{l}
A_{e} \exp \left(-\lambda \sqrt{C+\left(t-t_{\text {peak }}\right)^{2}}\right), t<t_{\text {peak }} \\
A_{q} \exp _{q}\left(-\lambda_{q} \sqrt{C+\left(t-t_{\text {peak }}\right)^{2}}\right), t \geq t_{\text {peak }}
\end{array}\right.
$$




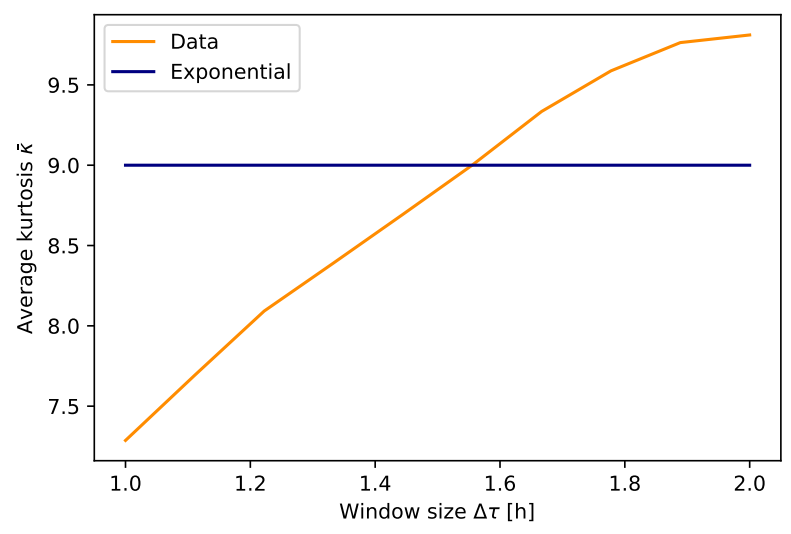

FIG. 8. The average kurtosis $\bar{\kappa}$ of the data set is plotted as a function of the time window $\Delta \tau$ in hours (blue). The intersection between the horizontal line at $\bar{\kappa}=9$ (the kurtosis of an exponential distribution) and the $\bar{\kappa}$ vs $\Delta t$ curve gives the optimal value for $\Delta t$; we find $T \approx 1.55$ hours.
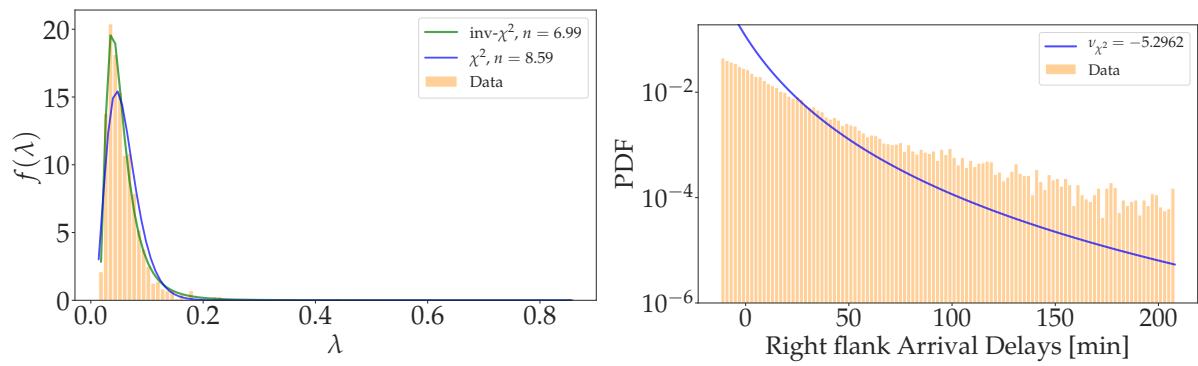

FIG. 9. Applying superstatistics leads to consistent results. Left: We extract the distribution of local exponents and compare them to a $\chi^{2}$ and inverse $\chi^{2}$ fit (based on the method of least squares). Right: Using the previously derived $\chi^{2}$ distribution, we again derive a $q$-exponential with right exponent $\nu_{\chi^{2}} \approx-5.296$, compared to the fitted one of $\nu_{\mathrm{MLE}} \approx-5.371$. Hence, while the low delays are not perfectly matched by the $\chi^{2}$-based $q$-exponential, the tail behavior is decently captured.

with $\exp _{q}(t)=(2-q) \lambda_{q}\left[1+(q-1) \lambda_{q} t\right]^{\frac{1}{1-q}}$ being the $q$-exponential function. Here, $A_{e}$ and $A_{q}$ are amplitudes, $C$ is a curvature parameter, describing the approximately Gaussian part in the center, $t_{\text {peak }}$ is the delay at the peak of the delay distribution, where we split into left and right flank and $t$ are the delay values, see Methods for fitting details.

The resulting fit is a smooth function, covering the full delay range, see Fig. 10. Since the new curvature parameter $C$ also influences the general shape, the new values for $q$ and $\lambda$, now named $\tilde{q}$ and $\tilde{\lambda}$, are slightly different from the ones solely focusing on the tails (empirically we tend to observe a slight reduction in $\lambda$ and increase in $q$ ). Still, the general observations using the delay indices and comparing airlines, such as in Figs. 4.6. remain mostly unchanged. Equation (5) provides an alternative approach to the three delay indices introduced so far. If one is interested in describing the full distribution as accurately as possible, we recommend using equation(5). Meanwhile, to compare performance of individual airlines or to obtain a general impression of the delay distribution, the three delay indices are a simplified framework, allowing easy and robust estimation and comparison. Finally, note that the full curve is not strictly a probability density function as we did not enforce that its integral equals one. While theoretically making it easier by reducing the number of parameters, that would make the fitting more difficult in practice as the integrals cannot be evaluated analytically by hand but only impose additional constraints during 
the fitting. Also note that our observed flight delays live on the finite interval [-100,210], whereas the fitting function is defined on $[-\infty, \infty]$, which makes the normalization outside the interval ambiguous.
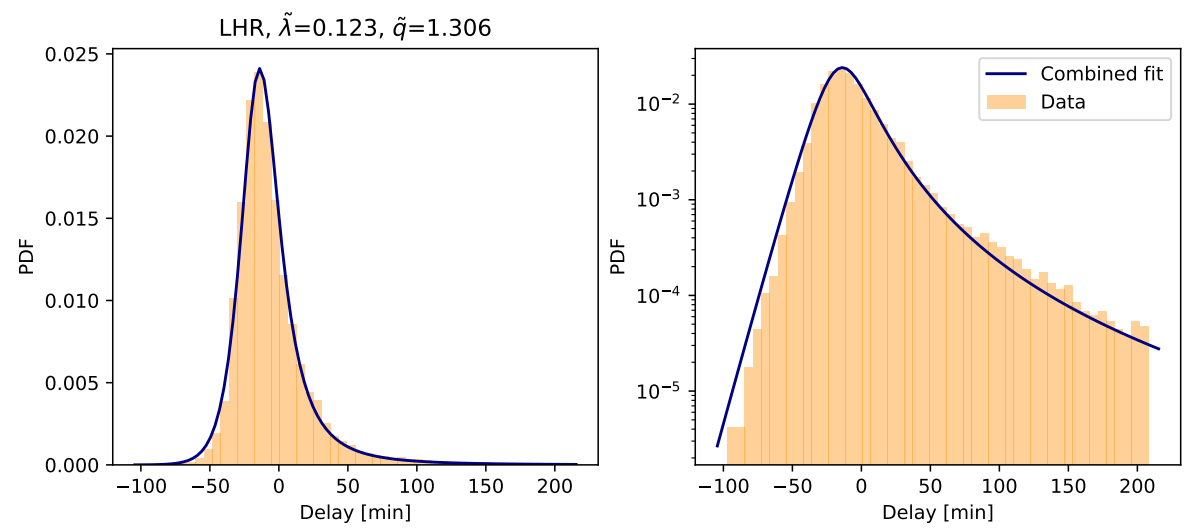

FIG. 10. Using the approximately Gaussian shape in the center, we smoothly combine left and right flank fits into one coherent fit of the full delay data set. To emphasize the quality of the fit, we display both a linear (left) and logarithmic (right) scale of the PDF for LHR.

\section{DISCUSSION AND CONCLUSIONS}

In summary, we have analysed a newly obtained data set of plane delays for various British airports, which contains tens of thousands of flights, aggregated over multiple months. This is a substantial improvement to some earlier studies which only investigated a few days of measurements and a couple of thousand flights, thereby greatly underestimating the contribution of the tails to the probability distribution 25. Interestingly, we find that all investigated airports and even individual airlines at each airport follow a qualitatively similar distribution, namely an approximately exponential decay on the left flank (of negative delays) and a slowly decaying power law on the right flank (of positive delays). To characterize these distributions and systematically compare airlines and airports, we have developed a framework to quantify delay performance. Critically, we do not only use the mean delay but also consider extreme events of both positive and negative delays via their respective flanks in the empirical probability distribution. Applying this newly developed framework, we find substantial differences between airlines serving short and long-distance routes.

We offer an explanation for the emerging power law on the right flank via superstatistics: The local $q$-exponential distribution with its heavy tails seems to arise from many superimposed exponential distributions. In particular, we identify the long time scale $T$ as approximately 1.5 hours, during which delays fall off exponentially. Comparing to other superstatistical results [21, 22, we note the relevance of both $\chi^{2}$-distributions and inverse- $\chi^{2}$-distributions for the scale parameter, similar to the ones observed in air pollution or cancer [14, 24, stressing again the universality of superstatistics. Finally, we propose a continuous function to capture the full delay statistics. While this introduces additional parameters and the superstatistical theory mentioned previously is no longer applicable, this fit does describe the full distribution with high accuracy.

Our framework of three delay indices to characterize flight delay distributions can be applied quite generally to measure the punctuality of flights, going beyond an analysis based on just the mean. Crucially, while airlines or airports might be able to "game" the system of mean delays, this is not possible with the left and right exponents. Companies could shift their flight schedule, i.e. announcing intentionally that flights will take longer than they do in practice, and thereby 
systematically record early arrivals so pushing their mean delay to negative values. However, such a procedure would still leave the remaining two indices (left and right exponent) untouched so that they provide a stable way of measuring performance.

One remarkable result is the impact of the global pandemic of COVID-19 on the delay statistics. Heathrow (LHR) under COVID-19 conditions (travel restrictions, quarantine upon arrival etc) displays an impressively low mean delay, while the left flank decay was mostly unchanged. Interestingly, LHR still experienced some heavily delayed flights during the COVID-19 pandemic, which leads to pronounced heavy tails towards the right and thereby a poor performance in the right exponent. These observations demonstrate that given fewer flights, the existing infrastructure is able to perform much better in some aspects (mean) than under "business-as-usual" conditions, while others (extreme delays) still can be improved. Aside from the upsides of COVID-19-related lockdown measures on air quality [26, 27] or $\mathrm{CO}_{2}$ emissions [28, we find that having fewer flights also improves delay statistics.

We have assumed throughout this article that negative delays are preferred by all passengers. However, some passengers might value arrival at exactly the predicted time more highly than arriving early. This would change the interpretation of the left index slightly: Instead of desiring low exponents, airlines and airports should aim for high exponents. Similarly, the absolute value of the delay should be zero, i.e. the arrival on time should be the default. Regardless of preference, the indices, as introduced, provide a sufficient framework to measure the delay performance.

In the future, we would like to apply our framework to delay statistics at other airports in different countries, and investigate how delays are related to geographical distance of the flights. In particular it would be interesting to see how our three indices differ between years, countries and so on. From a more fundamental perspective, we aim to further understand correlations in the flight delays. Preliminary indications from the British data are that on "typical" days correlations decay quickly but on some "exceptional" days (perhaps those where external factors affect many flights) the autocorrelation function can settle on a non-zero value for some time and many flights have long delays which contribute to the tail of the probability density function. Long-range temporal correlations and memory effects have been studied in many other physical and non-physical systems [29, 30]; modelling such effects here is challenging, since the build-up of delays at one airport may be influenced by earlier flights to and from completely different airports, but practically important since controlling the "cascading" of delays would lead to a significantly improved passenger experience.

\section{Acknowledgments}

This project has received funding from the European Union's Horizon 2020 research and innovation programme under the Marie Sklodowska-Curie grant agreement No 840825.

E.M., B.S., contributed equally. E.M., B.S., and C.B. conceived and designed the research. E.M. collected the data, E.M. and B.S. analysed the data and produced the figures. R.H. and all other authors contributed to discussing and interpreting the results and writing the manuscript.

The authors declare no competing interests. 
Here we explain the fitting procedure in more detail. We approximate the empirical distribution of the left flank, where negative delays are dominant, with an exponential distribution of the form

$$
p\left(t_{L} ; \lambda\right)=\lambda e^{-\lambda t_{L}}, \lambda>0 .
$$

As we have seen earlier, the observed distribution curves towards a Gaussian distribution around the peak value and thereby deviates from an exponential distribution. Hence, we restrict our fitting to values deviating from the central area as follows. Let $t_{\text {peak }}$ be the delay at which the distribution reaches its highest PDF value and $t_{\min }$ the smallest delay we observe. Then, we restrict our exponential fit to any delay falling in the interval $\left[t_{\text {min }}, t_{\text {peak }}-0.3\left|t_{\text {min }}-t_{\text {peak }}\right|\right]$, where $|\ldots|$ indicates the absolute value. Following this restriction, we define the left flank delay values as

$$
t_{L}=-t+t_{\text {peak }}-0.3\left|t_{\text {min }}-t_{\text {peak }}\right|, t \in\left[t_{\text {min }}, t_{\text {peak }}-0.3\left|t_{\text {min }}-t_{\text {peak }}\right|\right] .
$$

We now turn to the right flank of the empirical distribution, i.e. the data set that constitutes the majority of the positive delays. The $q$-exponential is much better at incorporating parts of the Gaussian central distribution on the right-hand side than the exponential distribution is on the left flank. Hence, we only exclude the smallest $10 \%$ of the data, i.e. we consider delays $t$ in the interval interval $\left[t_{\text {peak }}+0.1\left|t_{\max }-t_{\text {peak }}\right|, t_{\max }\right]$, where $t_{\max }$ is the highest delay observed. Hence the right-flank delays to be fitted are defined as

$$
t_{R}=t-t_{\text {peak }}-0.1\left|t_{\text {max }}-t_{\text {peak }}\right|, t \in\left[\left[t_{\text {peak }}+0.1\left|t_{\text {max }}-t_{\text {peak }}\right|, t_{\text {max }}\right] .\right.
$$

Our theoretical distribution choice is now a $q$-exponential

$$
p\left(t_{R} ; q, \lambda_{q}\right)=(2-q) \lambda_{q}\left[1+(q-1) \lambda_{q} t_{R}\right]^{\frac{1}{1-q}},
$$

with parameters $\lambda_{q}$ and $q$.

Note that both $t_{L}$ and $t_{R}$ are defined such that they start at 0 and continue towards positive values to keep the fitting functions easier.

These two functions (exponential and $q$-exponential) are fitted to the data using a maximum likelihood estimate (MLE), i.e. maximizing the Likelihood $L(\theta, \mathbf{x})$. Here, $\mathbf{x}$ indicates the data we wish to fit and $\theta$ the set of parameters that are being optimized. The likelihood of a parameter setting $\theta$ on a given one-dimensional data set $\mathbf{x}=x_{1}, x_{2}, \ldots, x_{N}$ is computed as

$$
L(\theta, \mathbf{x})=\prod_{i=1}^{N} p\left(x_{i}, \theta\right),
$$


with probability density function $p\left(x_{i}, \theta\right)$, dependent on the parameters $\theta$. Technically, we carry out the MLE using the scipy.stats module in python with custom PDFs, see also Code availability (below) for a link to the code.

Fitting the smooth combined function

To obtain a smooth fit, combining both flanks, we employ the following procedure. We first estimate the exponential decay rate $\lambda$ based on the lowest $70 \%$ of negative delays, then estimate $q$ and the $q$-exponential decay rate $\lambda_{q}$ based on almost the full right-hand side of the histogram. This is identical to the procedure for the individual flanking fits. Next, we estimate the central curvature $C$, which we assume to be identical for both intervals, and the amplitudes $A_{e}$ and $A_{q}$, as well as $\lambda_{q}$ using least squares fitting. While carrying out this least-square fit, we also allow the parameters $q$ and $\lambda$ to vary up to $10 \%$ from the MLE-optimal value determined earlier. The reason to allow any variance is to ensure a continuous fit and we want to keep the change from the optimal MLE parameters small, hence, we restrict it to $10 \%$. Technically, we use the scipy.stats module to perform the MLE fits and the least square fit; continuity is ensured using constraints in the symfit package.

\section{Airline data}

In Figs. 4 and 5 we compared several airlines. Let us briefly list how many flights we analysed to derive our delay indices: For the short-distance airlines 'Wizz Air': 2428, 'easyJet': 15449, 'Ryanair': 13488, 'Vueling': 1034, 'Jet2': 1215 and for the other airlines we have 'Iberia': 12892, 'British Airways': 38257, 'Aer Lingus': 7331, 'Finnair': 8560, 'American Airlines': 23119, 'Air Canada': 7247, 'United Airlines': 6797, 'Japan Airlines': 5966, 'Qatar Airways': 5935. For all airlines we have at least 1000 flights and often several thousand flights.

The original data of airport arrivals has been uploaded to an open repository: https://osf. io/snav9/?view_only=74c3a41eab9345aa9bae2b8bc322775c. All data that support the results presented in the figures of this study are available from the authors upon reasonable request.

\section{Code availability}

Python code to reproduce figures, perform the fits and extract the delay indices, is also uploaded here: https://osf.io/snav9/?view_only=74c3a41eab9345aa9bae2b8bc322775c.

[1] M. M. Hakim and R. Merkert, Journal of Transport Geography 56, 120 (2016).

[2] J. G. Brida, P. D. Monterubbianesi, and S. Zapata-Aguirre, World Review of Intermodal Transportation Research 7, 310 (2018).

[3] P. Suau-Sanchez, A. Voltes-Dorta, and N. Cugueró-Escofet, Journal of Transport Geography (2020).

[4] H. Kuhn, C. Falter, and A. Sizmann, in Proceedings of the 3rd CEAS AirESPace Conference and 21st AIDAA Congress, Venice, Italy (2011) pp. 1249-1259.

[5] M. Efthymiou, E. T. Njoya, P. L. Lo, A. Papatheodorou, and D. Randall, Journal of Aerospace Technology and Management 11 (2019). 
[6] J. J. Rebollo and H. Balakrishnan, Transportation Research Part C: Emerging Technologies 44, 231 (2014).

[7] J. M. Rosenberger, A. J. Schaefer, D. Goldsman, E. L. Johnson, A. J. Kleywegt, and G. L. Nemhauser, Transportation Science 36, 357 (2002).

[8] E. Mueller and G. Chatterji, in AIAA's Aircraft Technology, Integration, and Operations (ATIO) 2002 Technical Forum (2002) p. 5866.

[9] G. Gui, F. Liu, J. Sun, J. Yang, Z. Zhou, and D. Zhao, IEEE Transactions on Vehicular Technology 69, 140 (2019).

[10] M. Z. Li and M. S. Ryerson, Journal of Air Transport Management 75, 111 (2019).

[11] C. Beck, E. G. D. Cohen, and H. L. Swinney, Physical Review E 72, 056133 (2005).

[12] K. Briggs and C. Beck, Physica A: Statistical Mechanics and its Applications 378, 498 (2007).

[13] B. Schäfer, C. Beck, K. Aihara, D. Witthaut, and M. Timme, Nature Energy 3, 119 (2018).

[14] G. Williams, B. Schäfer, and C. Beck, Physical Review Research 2, 013019 (2020).

[15] R. Metzler, The European Physical Journal Special Topics 229, 711 (2020).

[16] UK Civil Aviation Authority, "UK Airports - Annual Statements of Movements, Passengers and Cargo -Table 09," (2019), [Online; accessed 10-September-2020].

[17] Kalyeena Makortoff , "Heathrow cargo flights rise 500\% as airport restyles itself as "vital airbridge,," [(2020), [Online; accessed 19-August-2020].

[18] S. Nižetić, International Journal of Energy Research 44, 10953 (2020).

[19] C. Tsallis, Journal of Statistical Physics 52, 479 (1988).

[20] EUROCONTROL, "Delays - three questions and many answers," (2018), [Online; accessed 10September-2020].

[21] C. Beck, Physical Review Letters 87, 180601 (2001).

[22] C. Beck and E. G. D. Cohen, Physica A: Statistical Mechanics and its Applications 322, 267 (2003).

[23] J. Weber, M. Reyers, C. Beck, M. Timme, J. G. Pinto, D. Witthaut, and B. Schäfer, Scientific Reports 9, 1 (2019).

[24] L. L. Chen and C. Beck, Physica A: Statistical Mechanics and its Applications 387, 3162 (2008).

[25] M. V. Caccavale, A. Iovanella, C. Lancia, G. Lulli, and B. Scoppola, Journal of Air Transport Management 34, 116 (2014).

[26] A. M. Shrestha, U. B. Shrestha, R. Sharma, S. Bhattarai, H. N. T. Tran, and M. Rupakheti, EarthArxiv (2020).

[27] B. Schäfer, R. Verma, A. Giri, H. He, S. Nagendra, M. Khare, and C. Beck, arXiv preprint arXiv:2007.00755 (2020).

[28] C. Le Quéré, R. B. Jackson, M. W. Jones, A. J. Smith, S. Abernethy, R. M. Andrew, A. J. De-Gol, D. R. Willis, Y. Shan, J. G. Canadell, et al., Nature Climate Change , 1 (2020).

[29] G. Rangarajan and M. Ding, eds., Processes with Long-Range Correlations: Theory and Applications, Lecture Notes in Physics, Vol. 621 (Springer-Verlag, Berlin Heidelberg, 2003).

[30] J. Beran, Y. Feng, S. Ghosh, and R. Kulik, Long-Memory Processes: Probabilistic Properties and Statistical Methods, berlin heidelberg ed. (Springer-Verlag, 2013). 


\section{Figures}

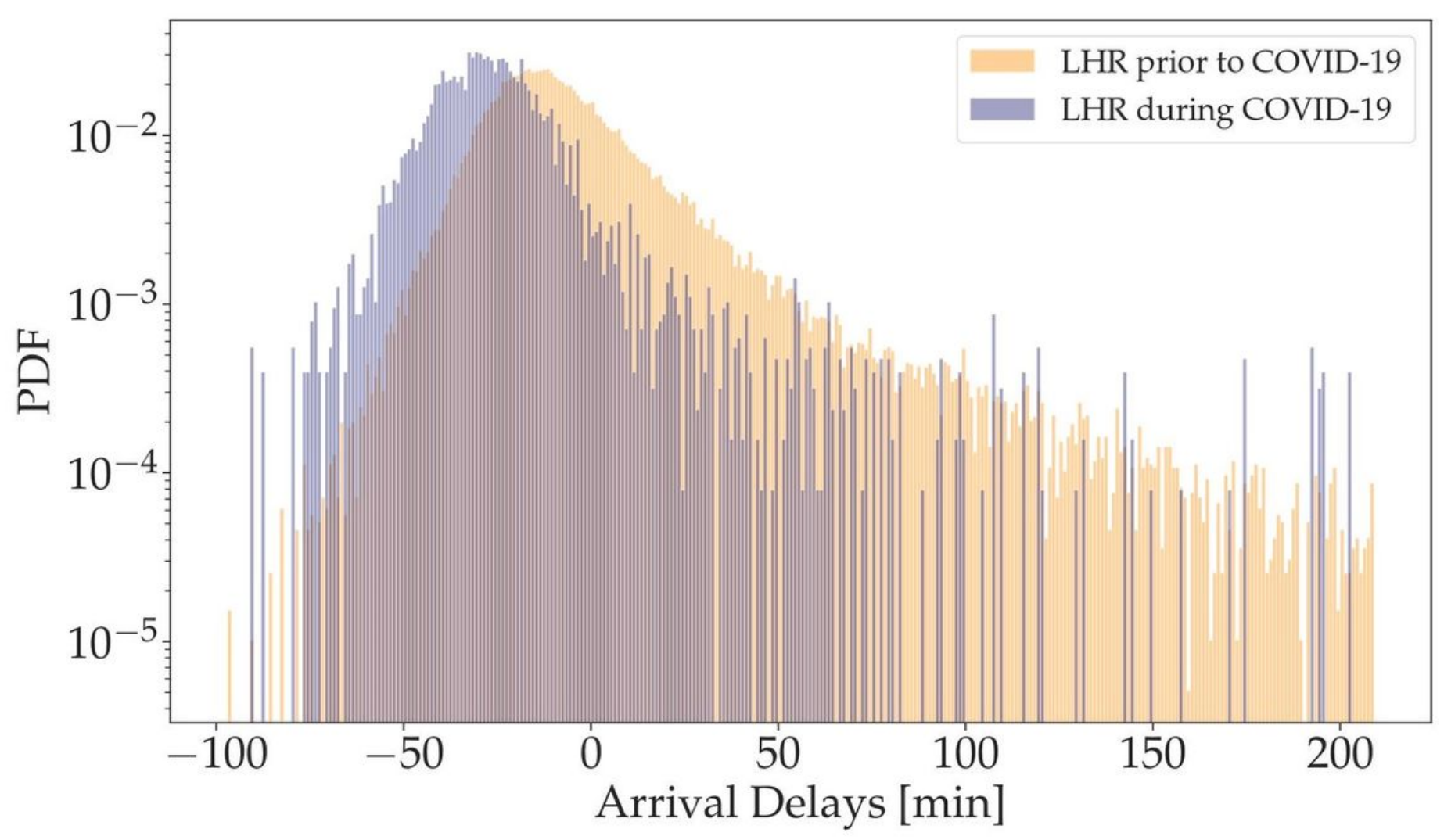

\section{Figure 1}

Flight delays follow a broad distribution with large negative and positive delays. We display LHR delay histograms prior to and during the COVID-19 pandemic, both normalized. As the COVID-19 LHR data set is significantly smaller in size, compared to the regular LHR data set, it contains many gaps, where no data were recorded. The COVID-19 data set is significantly shifted towards the left (smaller delays) as compared to the pre-pandemic time.
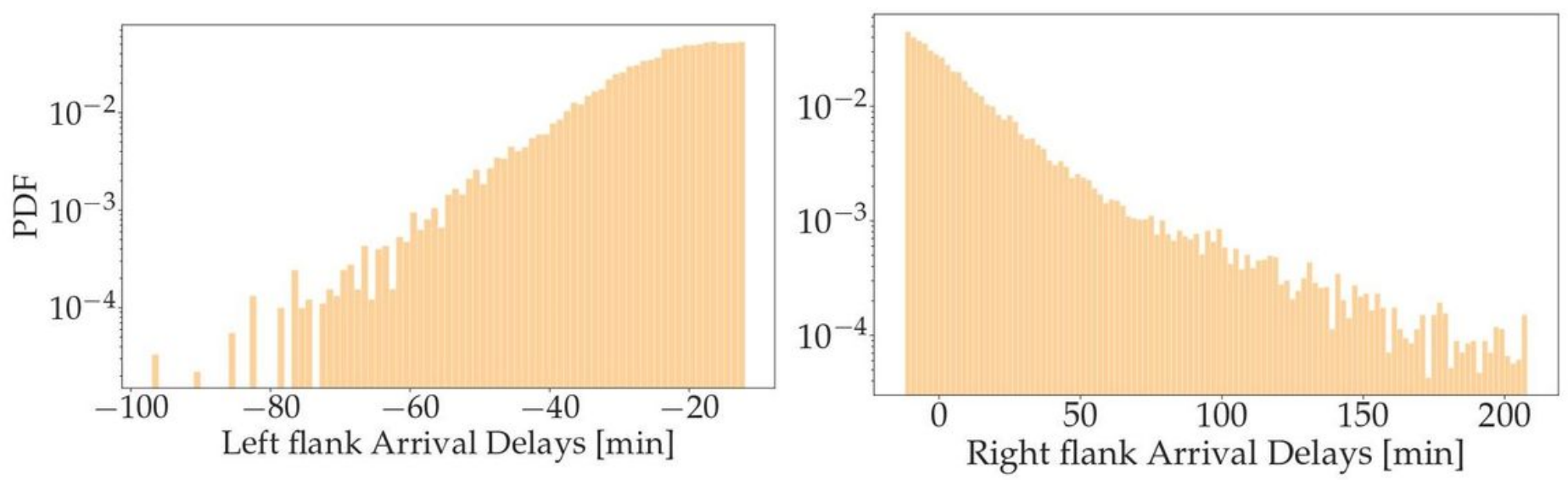
Figure 2

Splitting the full distribution at the peak leads to two easier-to-fit flanks. Left: Negative delays decay approximately linearly in the log-scale and thereby suggest an exponential fit (1) Right: Positive delays display substantial heavy tails and thereby suggest the usage of a q-exponential function (2).

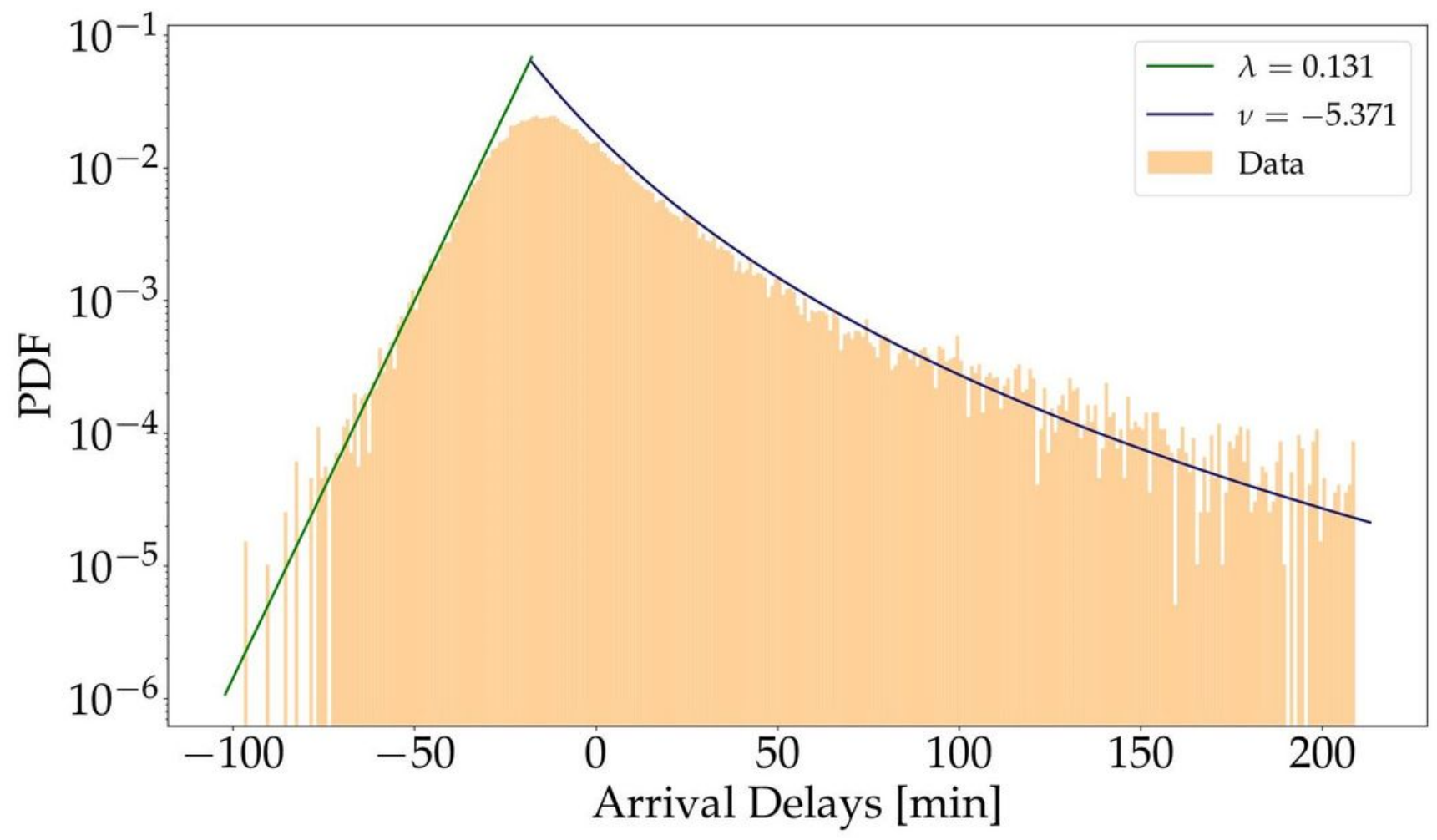

Figure 3

Exponential (green) and q-exponential (blue) theoretical distributions capture the empirical distribution. The fits are obtained via the MLE method, see Methods for fitting details. To complement the overestimated "peak" (tent-like shape) we introduce the mean delay $\mu$ index. 


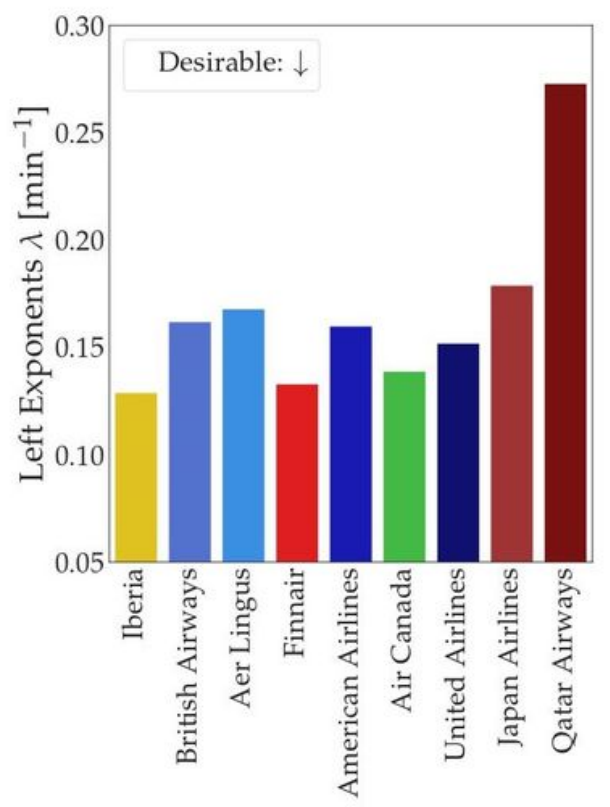

Airlines covering long-distance flights
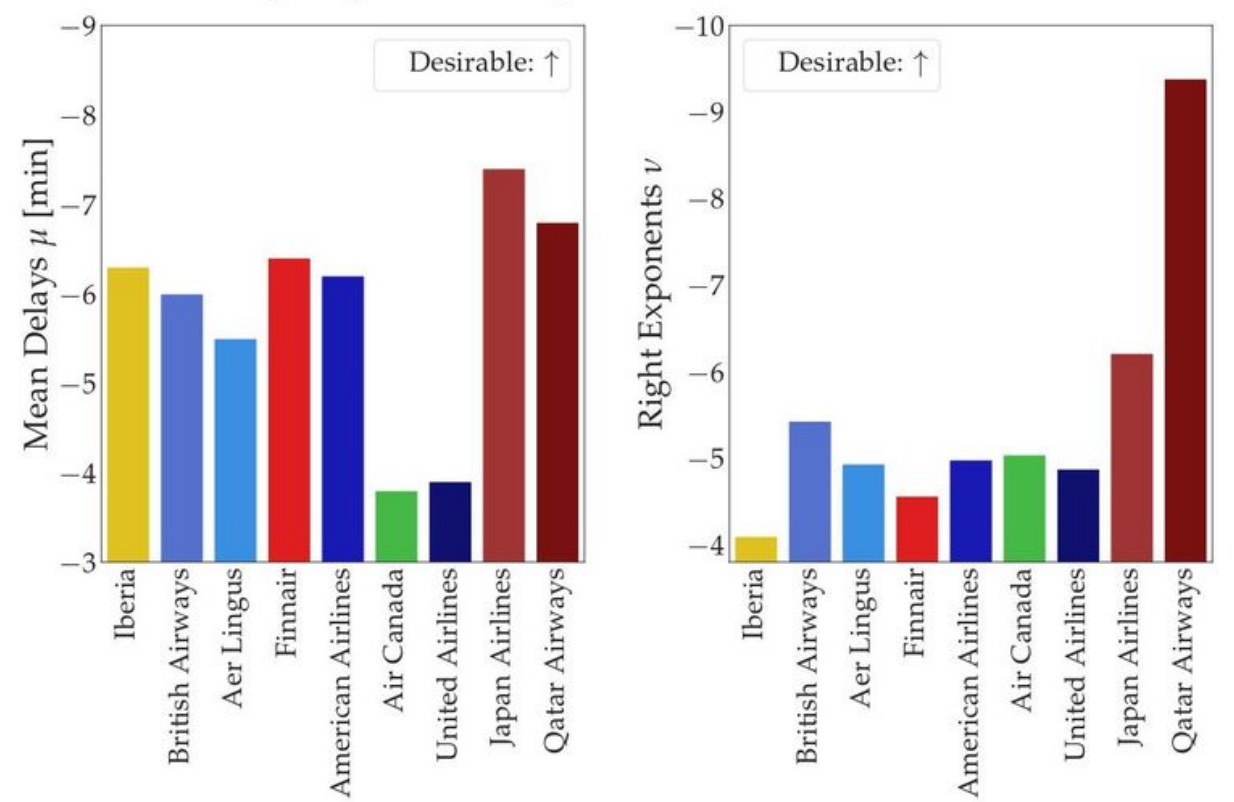

\section{Figure 4}

International airlines appear to differ substantially in their three delay indices. We plot the leftside (negative) delay exponential decay, right-side (positive) power-law delay decay and the mean delay. Arrows indicate whether a small or large value is desirable.

Airlines not covering long-distance flights
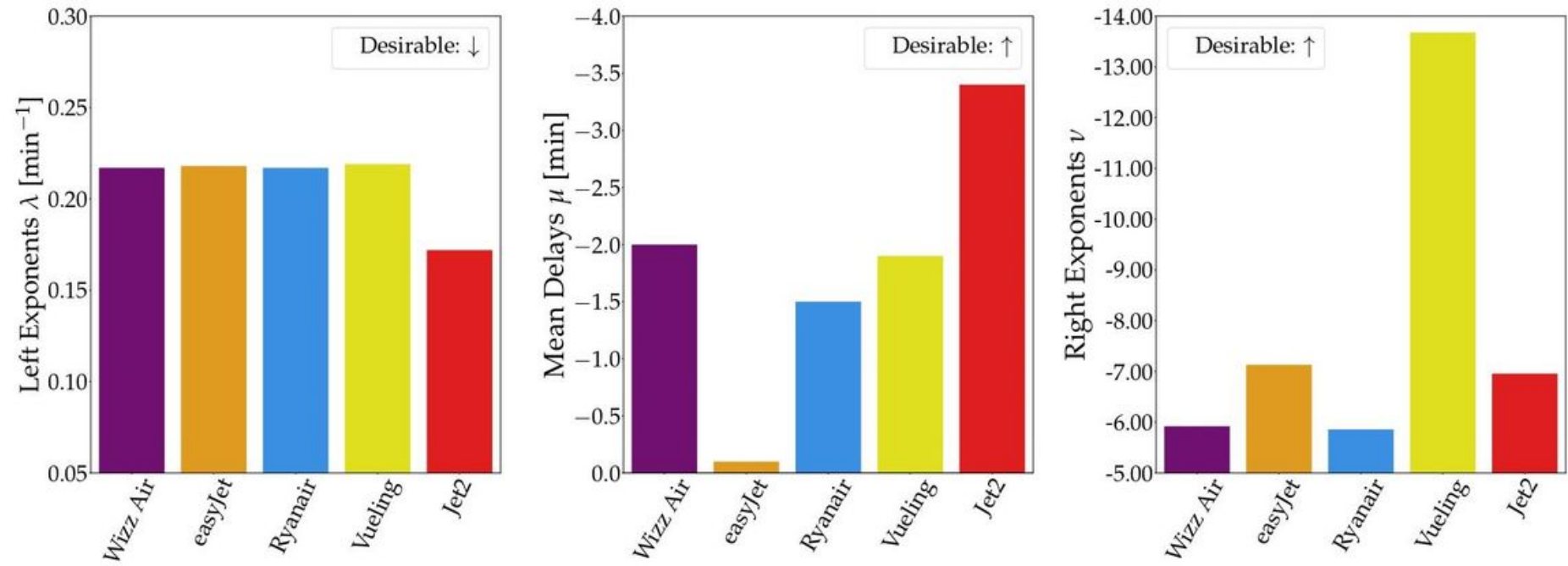

Figure 5

Delay indices for low-cost airlines not covering long-distance flights. Wizz Air, easyjet, Ryanair and Vueling share the largest $\lambda$ index (early arrivals). Jet2 has the smallest mean delay $\mu$ and Vueling is characterized by the smallest $v$ index (late arrivals). 
UK Airports Three Measures
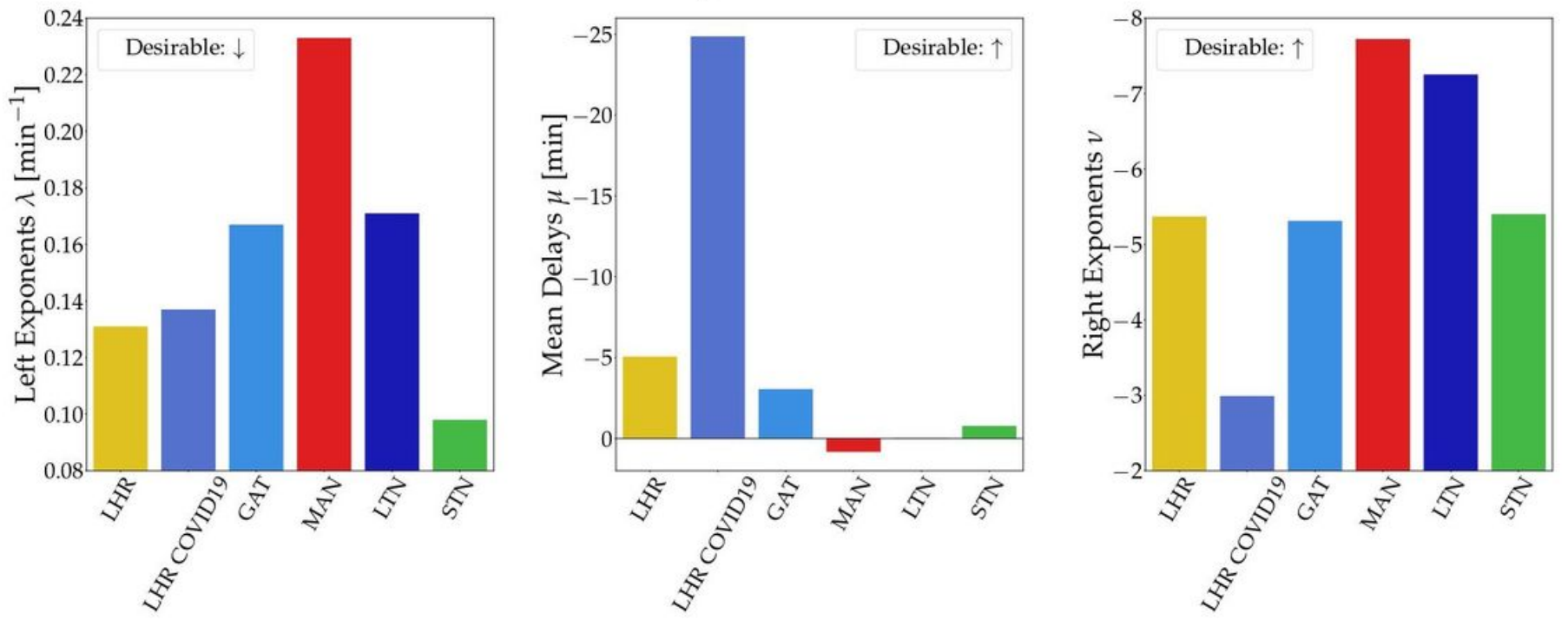

Figure 6

Airports appear to differ substantially in the three delay metrics. Airports that serve mostly domestic and European destinations, such as LTN and STN, behave differently from international airports such as LHR, GAT and MAN. 


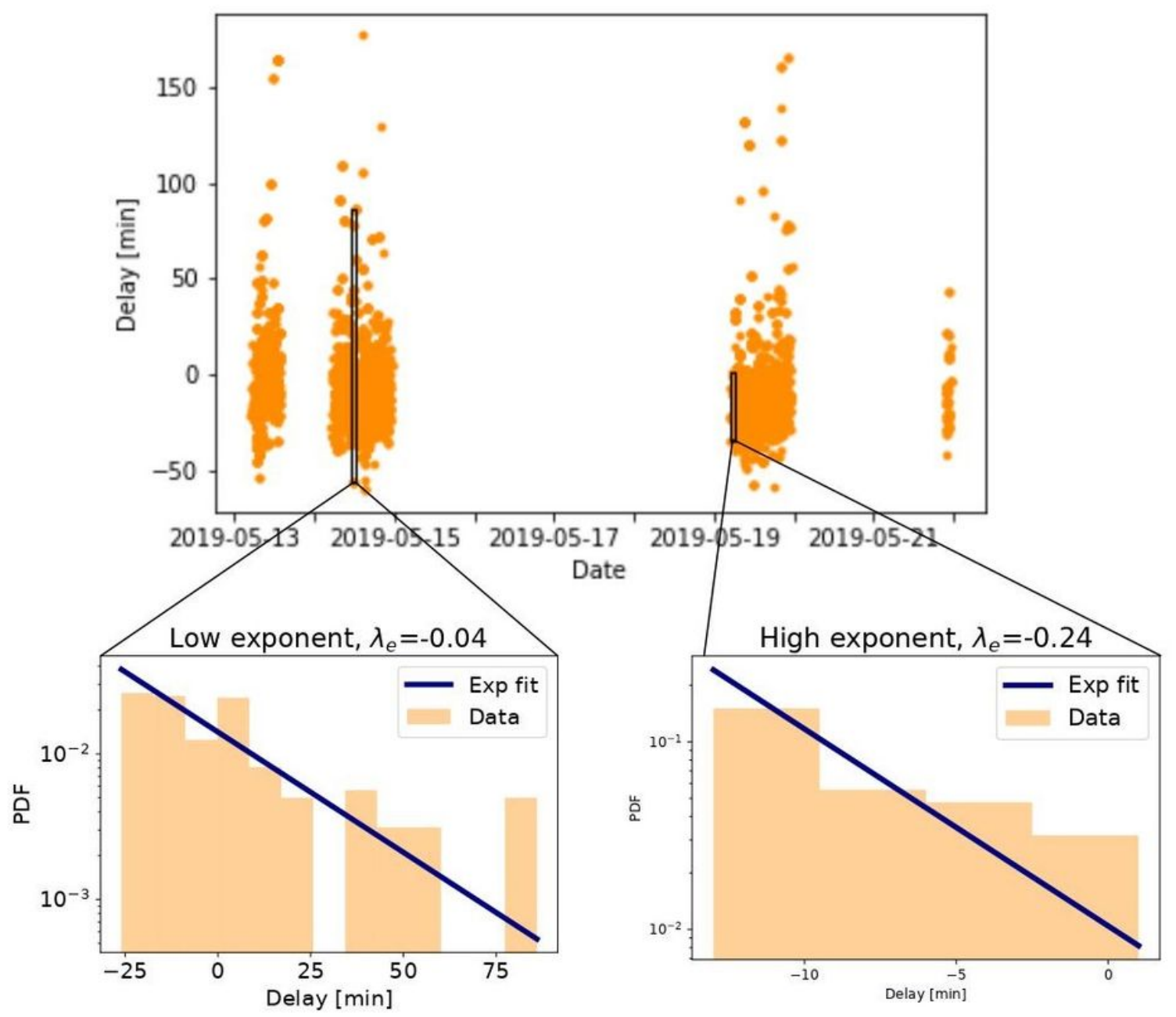

Figure 7

We analyse the full time series of plane delays and extract a time window during which we observe locally exponential distributions. These local distributions can decay slowly or fast, i.e. the rate $\lambda$ is fluctuating. 


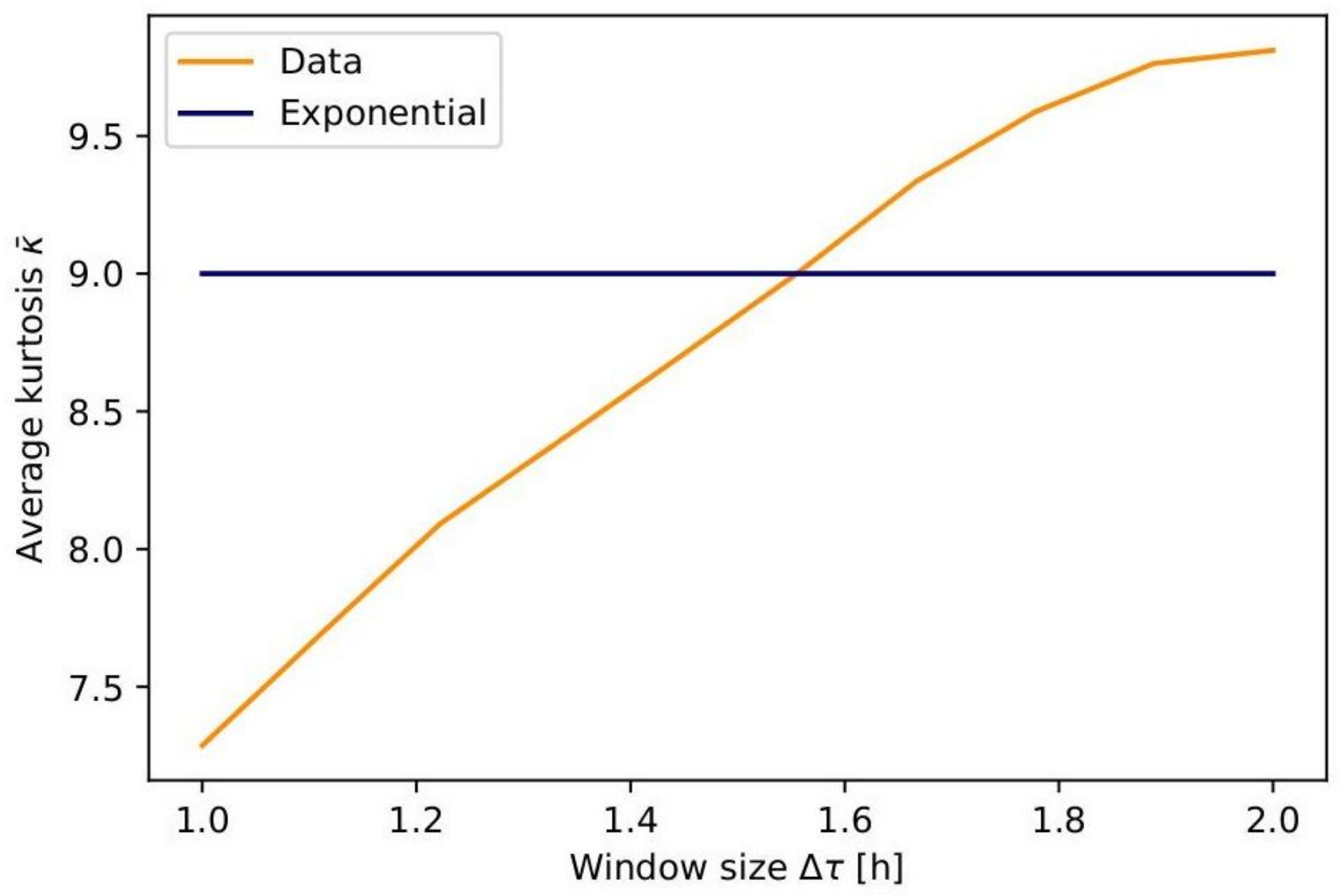

Figure 8

The average kurtosis ${ }^{-} \mathrm{K}$ of the data set is plotted as a function of the time window $\Delta \mathrm{t}$ in hours (blue). The intersection between the horizontal line at ${ }^{-} \mathrm{K}=9$ (the kurtosis of an exponential distribution) and the ${ }^{-} \mathrm{K}$ vs $\Delta \mathrm{t}$ curve gives the optimal value for $\Delta \mathrm{t}$; we find $\mathrm{T} \approx 1.55$ hours.
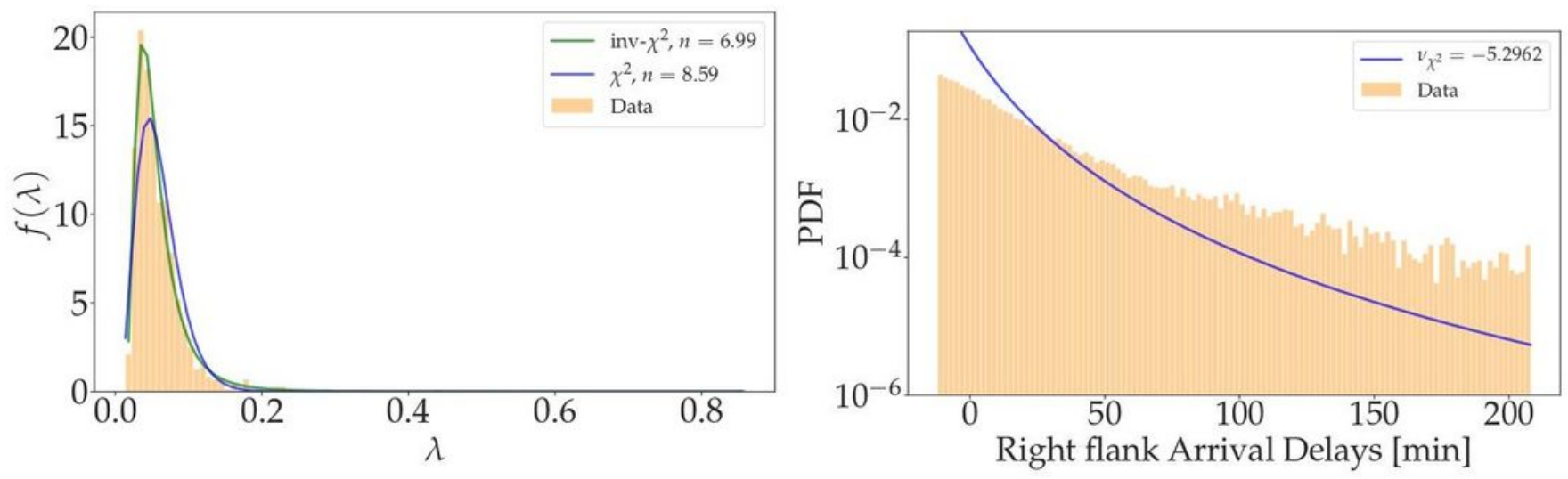

Figure 9 
Applying superstatistics leads to consistent results. Left: We extract the distribution of local exponents and compare them to a $\chi 2$ and inverse $\chi 2$ fit (based on the method of least squares). Right: Using the previously derived $\chi 2$ distribution, we again derive a q-exponential with right exponent $v \chi 2 \approx-5.296$, compared to the fitted one of $\mathrm{VMLE} \approx-5.371$. Hence, while the low delays are not perfectly matched by the $\chi 2$-based q-exponential, the tail behavior is decently captured.
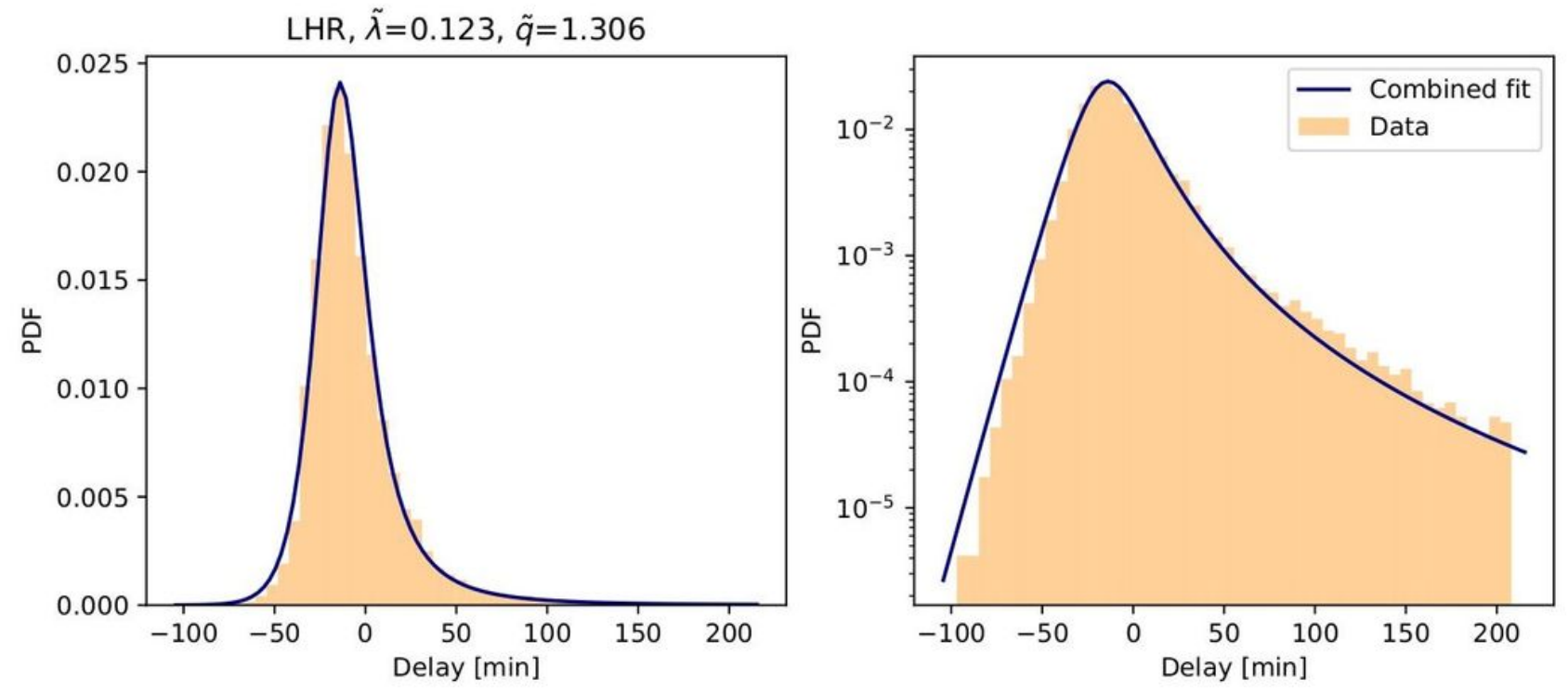

\section{Figure 10}

Using the approximately Gaussian shape in the center, we smoothly combine left and right flank fits into one coherent fit of the full delay data set. To emphasize the quality of the fit, we display both a linear (left) and logarithmic (right) scale of the PDF for LHR. 